Memories II International Congress Forests and Agroforestry for the 21st Century

Corresponding Author:

J. Criollo Barahona

Published: 21 January 2021

Production and Hosting by

Knowledge E

(c) J. Criollo Barahona et al. This article is distributed under the terms of the Creative Commons Attribution License, which permits unrestricted use and redistribution provided that the original author and source are credited.

\section{Determination of Physical and Mechanical Properties of Three-layer Boards of Teak (Tectona grandis), Seike (Cedrelinga catenaeformis) and Mascarey (Hieronima alchorneoides), Company PISMADE S.A., Riobamba Canton, Chimborazo Province}

\section{Determinación de Propiedades Físicas} y Mecánicas en Tableros Tricapa de Teca (Tectona grandis), Seike (Cedrelinga catenaeformis) y Mascarey (Hyeronima alchorneoides), Empresa PISMADE S.A., Cantón Riobamba, Provincia de Chimborazo

\author{
J. Criollo Barahona1, D. Román Robalino², E. Cabezas ${ }^{1}$, and E. Salazar \\ Castañeda ${ }^{2}$ \\ ${ }^{1}$ GIFOR Grupo de investigación forestal \\ ${ }^{2}$ Facultad de Recursos Naturales, Escuela Superior Politécnica de Chimborazo, Riobamba, \\ Ecuador
}

\section{Abstract}

The present investigation aims to determine the physical and mechanical properties in threelayer boards of Teak (Tectona grandis), Seike (Cedrelinga catenaeformis) and Mascarey (Hieronima alchorneoides), from the PISMADE S.A. Company, Canton Riobamba, province of Chimborazo. The three specimens used for each of the species in each of the tests performed were worked on using the ASTM D143-94 standard for the tests of parallel compression, perpendicular compression and flexion, and the DIN-52182 standard, with the main variation in thickness measurements caused by the commercial use established by the company. Mechanical tests showed that Seike had the best results, being superior in two tests: perpendicular compression and flexion. Teak tests gave us results that were superior in the perpendicular compression test. Mascarey, unlike the two species mentioned above, had complications during the trials due to problems with the glue between layers.

Keywords: physical and mechanical properties of wood, three-layer boards.

\section{Resumen}

La presente investigación pretende: Determinar las propiedades físicas y mecánicas en tableros tricapa de Teca, Seike (Cedrelinga catenaeformis) y Mascarey (Hyeronima alchorneoides), Empresa PISMADE S.A., cantón Riobamba, provincia de Chimborazo; para lo cual se utilizaron 3 probetas por cada una de las especies en cada uno de los ensayos realizados, las mismas 
fueron trabajadas con base a la Norma ASTM D143-94 para las pruebas de compresión paralela, compresión perpendicular y flexión y la Norma DIN-52182, teniendo como principal variación en las medidas de espesor debido al uso comercial establecido por la empresa. Las pruebas mecánicas mostraron que Seike tuvo los mejores resultados al ser superior en dos ensayos: Compresión perpendicular y flexión. Los ensayos con Teca nos dieron como resultados que es superior en el ensayo de compresión perpendicular. Mascarey a diferencia de las dos especies mencionadas anteriormente tuvo complicaciones con los ensayos debido a problemas con el pegamento entre capas.

Palabras Clave: propiedades físicas y mecánicas de la madera, tableros tricapa.

\section{Introducción}

El hombre ha usado la madera desde tiempo inmemorial como combustible y para construir sus habitaciones. Durante miles de años hemos aprendido a utilizarla en muebles y construcción. El desarrollo de los productos a base de madera ha solventado muchos problemas y abierto la oportunidad de incrementar las aplicaciones. Lo que ha logrado la industria es reconstituir la madera de deshecho o de mala calidad para la producción de paneles de superficies grandes fáciles de usar con caras pulidas, sin defectos localizados y con gran homogeneidad y estabilidad [1]. Los tableros de madera maciza más habituales son los de 3 capas (tricapas) y los de 5 capas [2]. El Tablero Tricapa es un tablero de encofrar con tres capas de madera de abeto o pino (sello PEFC) encoladas entre sí [3], está formado por dos capas externas de láminas de madera con la dirección de la fibra paralela a la longitud del tablero y al menos una capa interior perpendicular a las capas externas. Los tableros multicapa, debido a su composición, tienen una mayor estabilidad que si fueran de una sola pieza y es parecida a la de los tableros contrachapados. Depende de los coeficientes de contracción volumétrica, radial y tangencial de la especie de madera y de la composición del tablero. Este material ofrece las mejores condiciones para una manipulación fácil y eficaz y cumple todas las exigencias estéticas y técnicas. La cuidadosa selección óptico-mecánica de las láminas garantiza que los tableros presenten un número mínimo de grietas así como un dibujo bonito y equilibrado [4]. La resistencia del tablero dependerá de la durabilidad natural o adquirida de la madera y de la calidad de encolado [2].

\section{Materiales y Métodos}

\subsection{Desarrollo del proyecto}

El proyecto se realizó en el cantón Riobamba, provincia de Chimborazo, en PISOMAD perteneciente a la empresa PISMADE S.A. ubicada en Av. Bolívar Bonilla, y Pasaje Manila, sector Parque Industrial. De igual manera se utilizaron los laboratorios de las Facultades de Mecánica y Recursos Naturales de la Escuela Superior Politécnica de Chimborazo. 


\subsection{Normas para ensayos en madera}

Los ensayos de compresión y flexión se realizarán en Base a la Norma ASTM D143-94.

1. El ensayo de compresión paralela se realiza con probetas de $5 \times 5 \times 20 \mathrm{~cm}$.

2. El ensayo de compresión perpendicular se realiza con probetas de $5 \times 5 \times 15 \mathrm{~cm}$.

3. El ensayo de Flexión se realiza con probetas de 2,5×2,5×41 cm.

Para el ensayo de propiedad física se realizará en base a la Norma DIN- 52182.

1. El ensayo de densidad se realiza con probetas de medidas de $3 \times 3 \times 10 \mathrm{~cm}$.

Las probetas tuvieron variación en cuanto a las normas en el espesor debido a las medidas que serán utilizadas por la empresa entre las diferentes capas.

\subsection{Análisis de datos}

\subsubsection{Resistencia de materiales}

La Resistencia de Materiales se ocupa del cálculo de los esfuerzos y deformaciones que se producirán debiendo garantizar el ingeniero que las deformaciones estén dentro de unos límites permisibles y obviamente que no se produzcan roturas [5]. El módulo de elasticidad (E), también llamado módulo de Young, es un parámetro característico de cada material que indica la relación existente (en la zona de comportamiento elástico de dicho material) entre los incrementos de tensión aplicados (ds) en el ensayo de tracción y los incrementos de deformación longitudinal unitaria (de) producidos [6].

$$
\sigma=\frac{P}{A} \text {. }
$$

Esfuerzo. Donde P es la carga y A el área del material.

$$
E=\tan \vartheta .
$$

Módulo de elasticidad. $\vartheta$ es el Angulo formado en la gráfica realizada de carga versus la deformación.

\section{Resultados y Discusión}

\subsection{Ensayo de compresión paralela}

Los datos obtenidos nos muestran que Teca presenta que una mayor resistencia a la compresión obtenido valores superiores de resistencia en las tres probetas ensayadas, teniendo como valor máximo de resistencia de $148,41 \mathrm{~kg} / \mathrm{cm}^{2}$, Seike obtuvo una valoración en resistencia como valor más alto de $98,33 \mathrm{~kg} / \mathrm{cm}^{2}$ mientras en los ensayos con Mascarey se obtuvo los valores más bajos esto debido a fallas en cuento a pegamento entre las capas y teniendo como valor máximo de resistencia de $56,69 \mathrm{~kg} \mathrm{~cm}$, el MOE as alto obtenido fue de 25 119,43 por parte de Mascarey. 


\section{Table 1}

Ensayo de compresión paralela en probetas de tableros tricapa de Mascarey, Teca y Seike.

\begin{tabular}{lll} 
Probeta & Tipo \\
No. & \\
\hline 1 & $\begin{array}{l}\text { Mascarey (Hyeronima } \\
\text { alchorneoides) }\end{array}$ \\
\hline 2 & $\begin{array}{l}\text { Mascarey (Hyeronima } \\
\text { alchorneoides) }\end{array}$ \\
\hline 3 & $\begin{array}{l}\text { Mascarey (Hyeronima } \\
\text { alchorneoides) }\end{array}$ \\
\hline 4 & $\begin{array}{l}\text { Teca (Tectona grandis) } \\
\text { Teca (Tectona grandis) }\end{array}$ \\
\hline 6 & $\begin{array}{l}\text { Teca (Tectona grandis) } \\
\text { Seike (Cedrelinga } \\
\text { catenaeformis) }\end{array}$ \\
\hline 8 & $\begin{array}{l}\text { Seike (Cedrelinga } \\
\text { catenaeformis) }\end{array}$ \\
\hline 9 & $\begin{array}{l}\text { Seike (Cedrelinga } \\
\text { catenaeformis) }\end{array}$ \\
\hline
\end{tabular}

$\begin{aligned} & \text { Resistencia } \\ & \text { compresión } \\ & \text { paralela } \\ & \mathbf{K g} / \mathbf{c m}^{2} \\ & 49,90\end{aligned}$
51,02
$\mathbf{5 6 , 6 9}$
140,89
118,36
$\mathbf{1 4 9 , 4 1}$
67,55
85,39
98,33

\begin{tabular}{l}
$\begin{array}{l}\text { Resistencia } \\
\text { compresión } \\
\text { paralela }\end{array}$ \\
Promedio \\
$\mathbf{5 2 , 5 4}$ \\
\hline $\mathbf{1 3 6 , 2 2}$ \\
\hline $\mathbf{8 3 , 7 6}$ \\
\hline
\end{tabular}

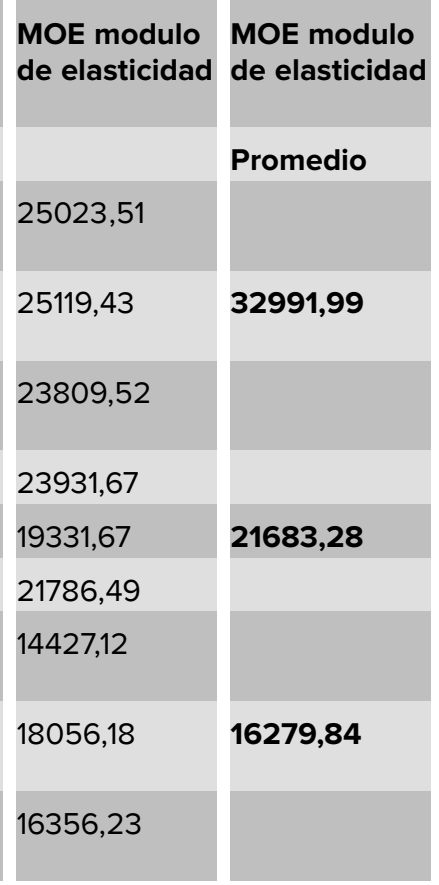

\section{Resistencia de Teca (Carga vs Deformación)}

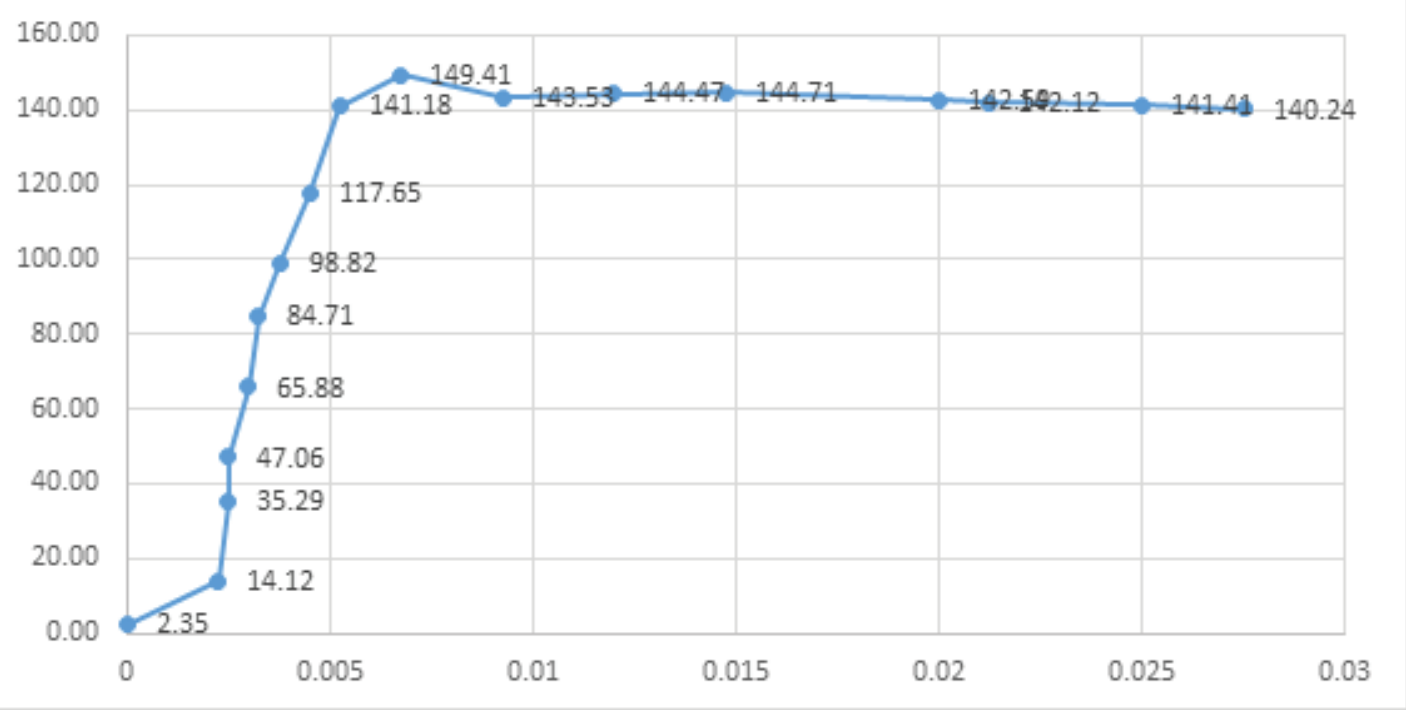

Figure 1

Grafica del comportamiento de Teca mediante carga aplicada en kg y deformación en cm.

\subsection{Ensayo de compresión perpendicular}

El ensayo realizado mostro que Seike tiene una resistencia a la compresión perpendicular superior con un valor máximo de $286,95 \mathrm{~kg} / \mathrm{cm}^{2}$, mientras Teca y Mascarey 
obtuvieron valores menores siendo Teca el que obtuvo el valor más cercano a Seike con un Valor de $281 \mathrm{~kg} / \mathrm{cm}^{2}$ y finalmente Mascarey con un valor de $213,37 \mathrm{~kg} / \mathrm{cm}^{2}$.

\section{Table 2}

Ensayo de compresión perpendicular en probetas de tableros tricapa en Mascarey, Teca y Seike.

\begin{tabular}{ll|l|l|l|l|} 
Probeta & Tipo & $\begin{array}{l}\text { Número de } \\
\text { capas }\end{array}$ & $\begin{array}{l}\text { Resistencia a } \\
\text { compresión } \\
\text { perpendicular } \\
\text { Kg/cm }\end{array}$ & $\begin{array}{l}\text { MOE (modulo de } \\
\text { elasticidad) }\end{array}$ & $\begin{array}{l}\text { MOE (modulo de } \\
\text { elasticidad) }\end{array}$ \\
\hline No. & Mascarey & 3 & 207,9 & 7074,19 & Promedio \\
\hline 1 & Mascarey & 3 & $\mathbf{2 1 3 , 3 7}$ & 10027,00 & $\mathbf{8 9 9 0 , 9 5}$ \\
\hline 2 & Mascarey & 3 & 212 & 9871,66 & \\
\hline 3 & Teca & 3 & $\mathbf{2 8 1}$ & 5768,35 & $\mathbf{7 0 7 3 , 1 0}$ \\
\hline 4 & Teca & 3 & 188 & 9721,42 & \\
\hline 5 & Teca & 3 & 164 & 5729,54 & $\mathbf{1 3 9 9 3 , 6 2}$ \\
\hline 6 & Seike & 3 & 245,21 & 14849,58 & \\
\hline 7 & Seike & 3 & 266,07 & 11363,61 & \\
\hline 8 & Seike & 3 & $\mathbf{2 8 6 , 9 5}$ & 15767,67 & \\
\hline 9 & & & & &
\end{tabular}

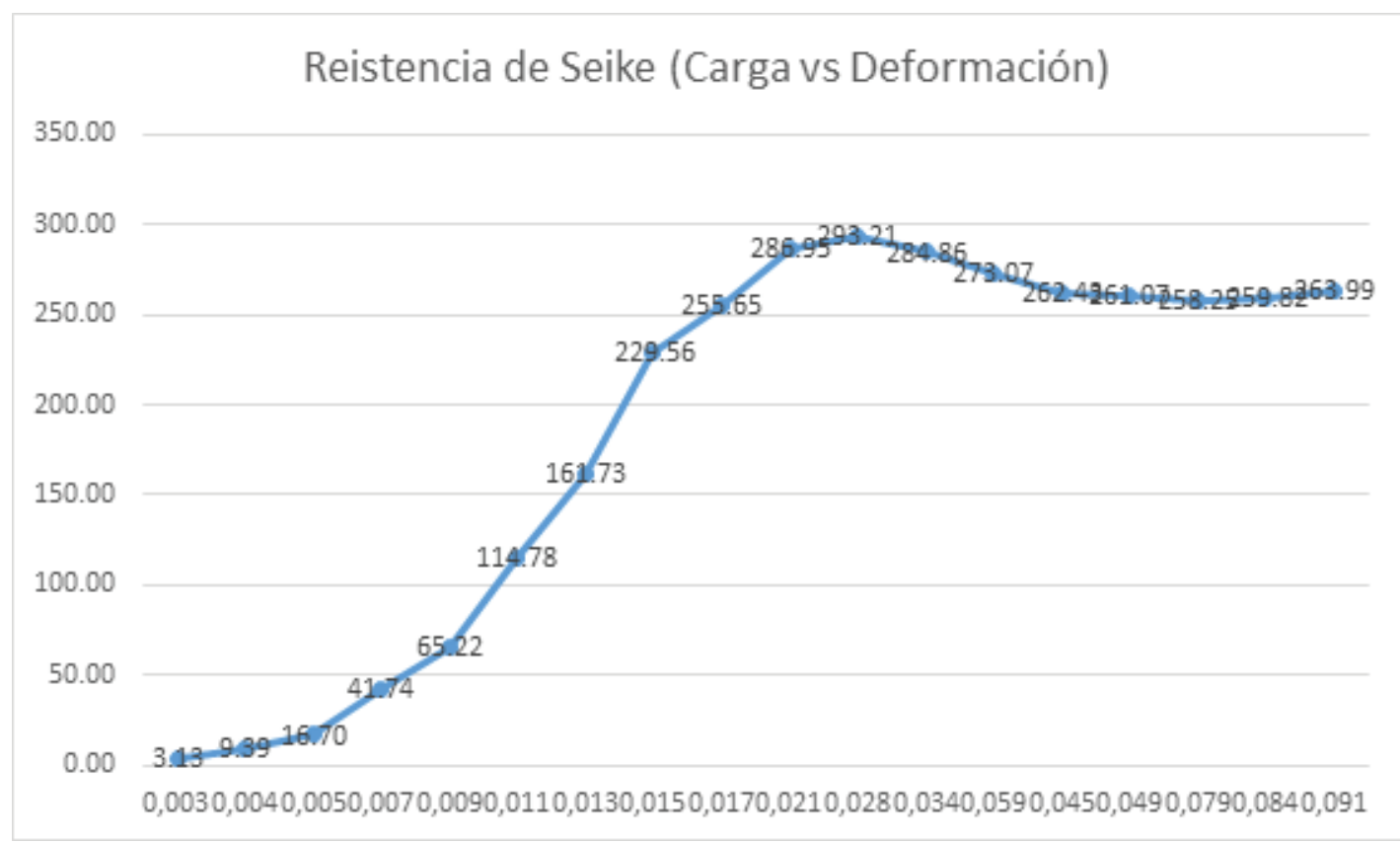

Figure 2

Resistencia de Seike en el ensayo de compresión perpendicular donde obtuvo el valor más alto de todas las especies. 


\subsection{Ensayo de flexión}

El mejor resultado de resistencia lo obtuvo Seike con un valor máximo de $305 \mathrm{~kg} / \mathrm{cm}^{2}$ (Figura 3) seguido de Teca con un valor de $230 \mathrm{~kg} / \mathrm{cm}^{2}$. En Mascarey se volvió a evidenciar problemas con el pegamento entre capas provocando que la resistencia obtenida nos sea la óptima. EI MOE más alto obtenido es por parte de Teca con 1190,68 $\mathrm{kg} / \mathrm{cm}^{2}$ (Tabla 3).

\section{Table 3}

Ensayo de flexión en probetas de tableros tricapa de Mascarey, Teca y Seike.

\begin{tabular}{|c|c|c|c|c|c|c|}
\hline Probeta & Tipo & $\begin{array}{l}\text { Longitud } \\
\text { inicial }\end{array}$ & $\begin{array}{l}\text { Número de } \\
\text { capas }\end{array}$ & $\begin{array}{l}\text { Resistencia a } \\
\text { la flexión }\end{array}$ & $\begin{array}{l}\text { MOE (modulo } \\
\text { de } \\
\text { elasticidad) }\end{array}$ & $\begin{array}{l}\text { MOE (modulo } \\
\text { de } \\
\text { elasticidad) }\end{array}$ \\
\hline No. & & $\mathrm{cm}$ & & $\mathrm{Kg} / \mathrm{cm}^{2}$ & & Promedio \\
\hline 1 & Mascarey & 35,5 & 3 & 100 & 828,73 & 607,07 \\
\hline 2 & Mascarey & 35,5 & 3 & 30 & 498,05 & \\
\hline 3 & Mascarey & 35,5 & 3 & 70 & 494,44 & \\
\hline 4 & Teca & 35,5 & 3 & 200 & 1000,1 & 1114,94 \\
\hline 5 & Teca & 35,5 & 3 & 170 & 1190,68 & \\
\hline 6 & Teca & 35,5 & 3 & 230 & 1154,03 & \\
\hline 7 & Seike & 35,5 & 3 & 300 & 974,11 & 974,46 \\
\hline 8 & Seike & 35,5 & 3 & 305 & 1111,27 & \\
\hline 9 & Seike & 35,5 & 3 & 250 & 837,99 & \\
\hline
\end{tabular}

\section{Ensayo de Flexión de Seike}

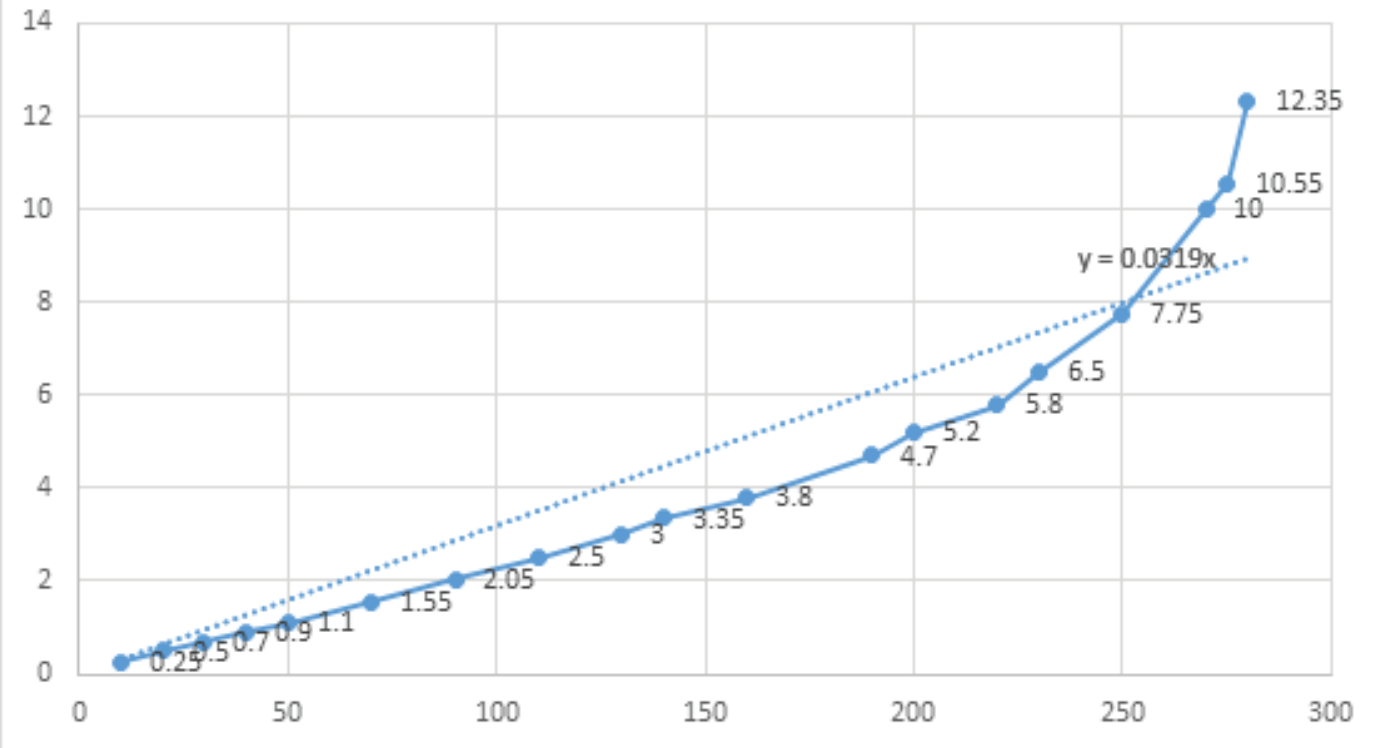

Figure 3

Flexión en seike con el mejor resultado obtenido en el ensayo. 


\subsection{Resistencia características de los tableros}

Como se observa en la Tabla 4 las características obtenidas en estudios de modelos de plancha en tableros multicapa de 3 y 5 capas, realizadas por la Empresa Binderholz $\mathrm{GmbH}$ muestras resultados similares obteniendo valores de resistencia de tableros tricapa entre $23,8 \mathrm{~N} / \mathrm{mm}^{2}$ y $37,0 \mathrm{~N} / \mathrm{mm}^{2}$, las pruebas fueron realizados en tableros de 3 capas con diferente grosor nominal (Tabla 4), utilizando material como abetos y Pino en la parte central de los tableros, por otra parte los ensayos realizados muestran que las resistencia en las probetas realizadas se obtuvieron los siguientes valores $9,61 \mathrm{~N} / \mathrm{mm}^{2}$, $14,61 \mathrm{~N} / \mathrm{mm}^{2}, 27,55 \mathrm{~N} / \mathrm{mm}^{2}, 28,13 \mathrm{~N} / \mathrm{mm}^{2}, 22,55 \mathrm{~N} / \mathrm{mm}^{2}$ y $29,90 \mathrm{~N} / \mathrm{mm}^{2}$.

\section{Table 4}

Valores de resistencia y de rigidez característica de tableros tricapa realizados por la Empresa Binderholz GmbH.

\begin{tabular}{|c|c|c|c|c|c|c|c|c|c|c|c|c|}
\hline \multirow[b]{2}{*}{$\begin{array}{l}\text { Grosor nominal } \\
(\mathrm{mm})\end{array}$} & \multicolumn{9}{|c|}{ Tablero de 3 capas } & \multicolumn{3}{|c|}{ Tablero de 5 capas } \\
\hline & 12 & 16 & 19 & 22 & 27 & 40 & 42 & 50 & 60 & 35 & 42 & 55 \\
\hline $\begin{array}{l}\text { Capas de } \\
\text { cobertura }(\mathrm{mm})\end{array}$ & 3,75 & 5,1 & 5,2 & 6,2 & 8,7 & 8,45 & 8,5 & 12,5 & 12,5 & 6,0 & 7,8 & 8,5 \\
\hline $\begin{array}{l}\text { Capas } \\
\text { intermedias } \\
(\mathrm{mm})\end{array}$ & & & & & & & & & & 8,6 & 8,6 & 15,0 \\
\hline $\begin{array}{l}\text { Capa central } \\
(\mathrm{mm})\end{array}$ & 4,5 & 5,8 & 8,6 & 9,6 & 9,6 & 23,1 & 25,0 & 25,0 & 35,0 & 5,8 & 9,2 & 8,0 \\
\hline \multicolumn{13}{|c|}{ Carga sobre la plancha } \\
\hline $\mathrm{fm}, 0$ & 37,0 & 34,9 & 31,6 & 30,3 & 28,7 & 24,4 & 23,8 & 26,3 & 24,2 & 21,9 & 23,1 & 20,5 \\
\hline $\mathrm{fm}, 90$ & 6,7 & 6,5 & 8,1 & 7,3 & 6,4 & 11,4 & 11,9 & 9,2 & 11,5 & 13,8 & 12,5 & 15,1 \\
\hline $\mathrm{Em}, 0$ & 11300 & 11400 & 10900 & 11000 & 11400 & 9700 & 9500 & 10500 & 9600 & 8700 & 9200 & 8200 \\
\hline $\mathrm{Em}, 90$ & 1000 & 900 & 1450 & 1350 & 900 & 2600 & 2800 & 1800 & 2700 & 3600 & 3150 & 4150 \\
\hline
\end{tabular}

Table 5

Tabla de características obtenidas en ensayos de tableros tricapa.

\begin{tabular}{|c|c|c|c|c|c|c|}
\hline Resistencia $\mathrm{Kg} / \mathrm{cm}^{2}$ & 98 & 149 & 281 & 286,95 & 230 & 305 \\
\hline Resistencia N/mm² & 9,61 & 14,61 & 27,55 & 28,13 & 22,55 & 29,90 \\
\hline
\end{tabular}

\subsection{Características comerciales para su aprovechamiento}

Las plantaciones de Cedrelinga catenaeformis alcanzan presenta rápido crecimiento en volumen, alcanzando $360 \mathrm{~m}^{3}$ ha ${ }^{1}$ a la edad de 9 años en la clase de sitio alta, y 368 $\mathrm{m}^{3}$ ha ${ }^{1}$ a la edad de 18 años en la clase de sitio medio. El incremento corriente anual máximo volumen culmina muy temprano, a los 3 años, alcanzando 36 y $56 \mathrm{~m}^{3}$ ha ${ }^{1}$ año ${ }^{1}$ en las clases de sitio medio y alto, respectivamente. El turno de rotación silvicultural se produce a los 9,7 y 5 años con crecimientos de 13, 28 y $44 \mathrm{~m}^{3}$ ha $^{1}$ año ${ }^{1}$, en las 
clases de sitio bajo, medio y alto, respectivamente [7]. En el país las plantaciones listo y preparado el terreno, se realiza la plantación a espaciamientos de $4 \times 4 \mathrm{~m}$ (densidad de 625 plantas/ha) o de $4 \times 3 \mathrm{~m}$ (densidad de 833 plantas/ha). En el país el turno previsto para esta especie se encuentra entre 15 y 25 años. El rendimiento es de 15 a 20 $\mathrm{m}^{3} /$ ha/año en plantaciones y de apenas $1 \mathrm{~m}^{3} /$ ha/año en bosque natural [8].

Teca es un árbol que alcanza alturas mayores a $30 \mathrm{~m}$ de altura y $80 \mathrm{~cm}$ de DAP, la rotación es generalmente de 120 años, con un ciclo de corta de unos 30 años, las plantaciones Listo y preparado el terreno, se realiza la plantación a espaciamientos de $4 \times 4 \mathrm{~m}$ (densidad de 625 plantas/ha) o de $4 \times 3 \mathrm{~m}$ (densidad de 833 plantas/ha). Los rendimientos son muy variables dependiendo de los suelos, 8 a $25 \mathrm{~m}^{3} / \mathrm{ha} / \mathrm{año} \mathrm{y}$ teniendo turnos de mayor de 18 años, la madera tiene más valor si es de mayor edad y madurez [9].

Hyeronima alchorneoides $0,63 \mathrm{~g} / \mathrm{cm}^{3}$, muy pesada y algunas veces la densidad va desde $0,5 \mathrm{~g} / \mathrm{cm}^{3}$. Las plantaciones se hace a una distancia inicial utilizado en plantaciones es de $3 \times 3 \mathrm{~m}$, con turnos de aprovechamiento de aproximadamente 30 años [10].

\subsection{Densidad de tableros tricapa formadas con tres especies}

\section{Table 6}

Tabla de características de densidad obtenidas en ensayos de tableros tricapa.

\begin{tabular}{|c|c|c|c|c|c|c|c|}
\hline Probeta & Tipo & Ancho & Espesor & Largo & Volumen & Peso & Densidad \\
\hline No. & & $\mathrm{cm}$ & $\mathrm{cm}$ & $\mathrm{cm}$ & $\mathrm{cm}^{3}$ & g & $\mathrm{g} / \mathrm{cm}^{3}$ \\
\hline 1 & Mascarey & 3 & 3 & 10 & 90 & 55,31 & 0,61 \\
\hline 2 & Mascarey & 3 & 3 & 10 & 90 & 54,64 & 0,61 \\
\hline 3 & Mascarey & 3 & 3 & 10 & 90 & 51,51 & 0,57 \\
\hline 4 & Teca & 3 & 3 & 10 & 90 & 54,46 & 0,61 \\
\hline 5 & Teca & 3 & 3 & 10 & 90 & 60,81 & 0,68 \\
\hline 6 & Teca & 3 & 3 & 10 & 90 & 54,79 & 0,61 \\
\hline 7 & Seike & 3 & 3 & 10 & 90 & 55,52 & 0,62 \\
\hline 8 & Seike & 3 & 3 & 10 & 90 & 58,16 & 0,65 \\
\hline 9 & Seike & 3 & 3 & 10 & 90 & 57,43 & 0,64 \\
\hline
\end{tabular}

\section{Conclusiones}

Los resultados obtenidos en los ensayos muestran que las probetas de Tableros Tricapa de Seike ( $C$. catenaeformis) es la especie con mayor resistencia en compresión perpendicular y flexión con $286,5 \mathrm{~kg} / \mathrm{cm}^{2}$ y $305 \mathrm{~kg} / \mathrm{cm}^{2}$, respectivamente y un módulo de elasticidad de $15.767,67 \mathrm{~kg} / \mathrm{cm}^{2}$ y $1111,27 \mathrm{~kg} / \mathrm{cm}^{2}$, además teniendo un comportamiento regular en el ensayo de compresión paralela con $98,33 \mathrm{~kg} / \mathrm{cm}^{2}$ y una densidad media de $0,63 \mathrm{~g} / \mathrm{cm}^{3}$. 
Los ensayos en probetas de Tableros Tricapa de Teca (Tectona grandis) muestran que la resistencia en compresión paralela es de 149,41 kg/ $\mathrm{cm}^{2}$ y MOE de 23.931,67 $\mathrm{kg} / \mathrm{cm}^{2}$, y en compresión perpendicular y flexión es de $281 \mathrm{~kg} / \mathrm{cm}^{2}$ y $230 \mathrm{~kg} / \mathrm{cm}^{2}$, respectivamente y una densidad media de $0,63 \mathrm{~g} / \mathrm{cm}^{3}$.

Las probetas de Tableros tricapa de Mascarey (Hyeronima alchorneoides), muestran su irregularidad debido a los problemas presentados con el pegamento entre capas, lo cual impidió obtener el valor más alto antes de la ruptura de sus fibras, obteniendo los siguientes resultados en compresión paralela, perpendicular y flexión: 56,69 kg/cm², $213,37 \mathrm{~kg} / \mathrm{cm}^{2}$ y $100 \mathrm{~kg} / \mathrm{cm}^{2}$ y una densidad media de $0,60 \mathrm{~g} / \mathrm{cm}^{3}$. El mejor módulo de elasticidad la obtuvo en la prueba de compresión paralela con un valor de 25.119,43 $\mathrm{kg} / \mathrm{cm}^{2}$ siendo superior a Teca y Seike.

\section{References}

[1] Fernández A. Evolución del mundo tecnologico de los tableros de madera [Evolution of the technological world of wooden boards]. Lourizan; 1993 [Recuperado 2019 marzo 18]. Spanish

[2] AITIM (Asociación de Investigación Técnica de las Industrias de la Madera). 2011 [Recuperado 2019 abril 25].

[3] Vargas G. Maderas Portu; 2012 [Recuperado 2019 Marzo 15]. Desponisble en: http://maderasportu. com/tienda/tableros/tablero-tricapa-encofrado/

[4] Binderholz. Tableros de madera maciza y para la Contrucción [Solid wood boards and for construction]. 2012 [Recuperado 2019 abril 10. Spanish

[5] Zalazar J. 2007 [Recuperado el 15 de Junio de 2019]. Desponsible en: http://www.bdigital.unal.edu.co/ 5855/1/jorgeeduardosalazartrujillo20072_Parte1.pdf

[6] Universitat Jaume I. 2014 Junio [Recuperado 2019 Julio 18]. Desponsible en: http://www.mecapedia. uji.es/modulo_de_elasticidad.htm

[7] Baluarte J, Alvarez J. (s.f.). Modelamiento del crecimiento de tornillo cedrelinga catenaeformis ducke en plantaciones en Jenaro Herrera [Growth modeling of cedrelinga catenaeformis ducke screw in plantations in Jenaro Herrera,Internet]. Perú: departamento de loreto; s.f [Recuperado 2019 Octubre 12]. 1-12 p. Disponsible en: http://www.iiap.org.pe/upload/Publicacion/PUBL1433.pdf. Spanish

[8] Vinueza M. 2012 Julio [Recuperado 2019 Julio 14]. Desponisble en: https://ecuadorforestal.org/ fichas-tecnicas-de-especies-forestales/ficha-tecnica-no-1-teca/

[9] Vinueza M. Ficha Técnica de Chuncho; 2012 Octubre [Recuperado 2019 Julio]. Desponsible en: https: //ecuadorforestal.org/noticias-y-eventos/ficha-tecnica-no-9-chuncho/

[10] Ramirez J. 1999 [Recuperado 2019 julio 8]. Disponsible en: http://www.itto.int/files/itto_project_db_ input/2017/Technical/pd8-92-5-3\{\%\}20rev2(F)\%20s_Rosita_S.pdf 